\title{
Características clínicas y epidemiológicas de la endocarditis infecciosa en el Hospital Nacional Dos de Mayo, 2014-2019
}

\author{
Clinical and epidemiological characteristics of infective endocarditis at Hospital \\ Nacional Dos de Mayo, 2014-2019
}

\author{
Carolina Ferrer Rodríguez ${ }^{1, a}$, Martha Fu Llampasi ${ }^{1, b}$, Nora Espíritu ${ }^{1,2, c}$, Alejandra Parhuana Bando ${ }^{2,3, d}$ \\ ${ }^{1}$ Facultad de Medicina, Universidad San Martín de Porres. Lima, Perú. \\ ${ }^{2}$ Hospital Nacional Dos de Mayo. Lima, Perú. \\ ${ }^{3}$ Facultad de Medicina, Universidad Nacional Mayor de San Marcos. Lima, Perú. \\ ${ }^{a}$ Estudiante de medicina, ORCID: https://orcid.org/0000-0002-2412-2323 \\ ${ }^{\mathrm{b}}$ Estudiante de medicina, ORCID: https://orcid.org/0000-0001-6067-6828 \\ CMédica pediatra, magister en salud pública. ORCID: https://orcid.org/0000-0002-6340-0285- \\ ${ }^{d}$ Médica internista, magister en medicina. ORCID: https://orcid.org/0000-0002-3969-6047-
}

An Fac med. 2020;81(4):404-9. / DOI: https://doi.org/10.15381/anales.v81i4.19503

\section{Correspondencia: \\ Carolina Milagros Ferrer Rodríguez carolinaferrer1810@gmail.com}

Recibido: 26 de enero 2021

Aprobado: 13 de abril 2021

Publicación en línea: 15 de abril 2021

Conflictos de interés: Los autores

declaran no tener conflictos de interés.

Fuente de financiamiento: Autofinanciado

Citar como: Ferrer C, Fu M, Espiritu N, Parhuana A. Características clínicas y epidemiológicas de la endocarditis infecciosa en el Hospital Nacional Dos de Mayo, 2014-2019. An Fac med. 2020;81(4):404-9. DOl: https://doi. org/10.15381/anales.v81i4.19503.

\section{Resumen}

Introducción. La endocarditis infecciosa (EI) es una enfermedad causada por la invasión de organismos patógenos al endotelio cardiaco. Objetivos. Determinar las características clínicas y epidemiológicas de la endocarditis infecciosa en el Hospital Nacional Dos de Mayo, 2014-2019, Lima-Perú. Métodos. Estudio observacional, descriptivo y retrospectivo. Se incluyeron a pacientes mayores de 18 años con diagnóstico de El según criterios de Duke modificados, que contaban con hemograma, hemocultivo y ecocardiograma. Resultados. Se estudiaron 26 pacientes; la edad promedio fue 46,96 \pm 15,6 años, predominó el sexo masculino (57,7\%), 15 procedian de Lima y el 50\% tenían como grado de instrucción secundaria completa. El $57,7 \%$ ( $n=15)$ tuvieron hemocultivo negativo, la sintomatología más frecuente fue soplo cardíaco $(67,6 \%)$, fiebre (26\%) y disnea $(23,4 \%)$, la complicación predominante fue la insuficiencia cardíaca $(61 \%)$. Las comorbilidades $(47,1 \%)$ fueron el principal factor de riesgo. La válvula mitral $(51,7 \%)$ fue la más comprometida. El régimen antibiótico vancomicina + ceftriaxona y vancomicina + gentamicina ambos con $19,2 \%$ fueron los más usados. El 88\% presentaron valores de amplitud de distribución eritrocitaria (ADE) > 14,4\%. De 10 que fallecieron, 9 tuvieron ADE > 14,4\%. Conclusiones. La El afectó principalmente a mayores de 50 años, de sexo masculino, con comorbilidades concomitantes. La ecocardiografía positiva junto a los criterios menores de Duke fueron predominantes. La mayoría presentó valores elevados de ADE que se relacionaron con la presencia de complicaciones y mortalidad, la cual fue alta.

Palabras clave: Endocarditis; Epidemiología Descriptiva; Complicaciones; Mortalidad (fuente: DeCS BIREME).

Abstract

Introduction. Infective Endocarditis (IE) is a disease caused by the invasion of pathogenic organisms into the cardiac endothelium. Objectives. To determine the clinical and epidemiological characteristics of Infectious Endocarditis in the Hospital Nacional Dos de Mayo, 2014-2019, Lima-Perú. Methods. Observational, descriptive and retrospective study. Patients older than 18 years with a diagnosis of IE according to modified Duke criteria, who had a complete blood count, blood culture, and echocardiogram were included. Results. 26 patients were studied; the average age was 46,96 $\pm 15,6$ years, males predominated (57,7\%), 15 came from Lima and 50\% had completed secondary education as a degree. $57,7 \%(n=15)$ had a negative blood culture, the most frequent symptoms were heart murmur $(67,6 \%)$, fever $(26 \%)$ and dyspnea $(23,4 \%)$, the predominant complication was heart failure $(61 \%)$. Comorbidities $(47,1 \%)$ were the main risk factor. The mitral valve $(51,7 \%)$ was the most compromised. The antibiotic regimen vancomycin + ceftriaxone and vancomycin + gentamicin, both with $19,2 \%$, were the most used. $88 \%$ had erythrocyte distribution width (RDW) values $>14,4 \%$. Of 10 who died, 9 had RDW $>14,4 \%$. Conclusions. IE mainly affects males over 50 years of age with concomitant comorbidities. Positive echocardiography together with the Duke minor criteria were predominant. Most presented high RDW values that were related to the presence of complications and mortality, which was high.

Keywords: Endocarditis; Epidemiology Descriptive; Complications; Mortality.

(source: MeSH NLM). 


\section{INTRODUCCIÓN}

La Endocarditis Infecciosa (EI) es una enfermedad causada por la invasión de organismos patógenos al endotelio cardiaco, que llegan por vía hematógena al revestimiento interior de las cámaras cardíacas donde fundamentalmente afectan a las válvulas generando vegetaciones, siendo estas las lesiones características de esta enfermedad ${ }^{(1)}$. Tienden a producir émbolos sépticos, abscesos y rotura tanto de las válvulas como de las cuerdas tendinosas ${ }^{(2)}$. Los elementos que intervienen en su patogenia son el daño endotelial, una fuente de bacteriemia y la virulencia del germen ${ }^{(2)}$. Afecta principalmente a pacientes con valvulopatías, portadores de válvulas protésicas, con cardiopatías congénitas, y que han sido sometidos a procedimientos de riesgo ${ }^{(3)}$. Debido a su alta mortalidad y a las complicaciones graves que conlleva, se considera una enfermedad de gran importancia para la salud pública a pesar de ser poco frecuente, con una incidencia de 3 a 9 casos por cada 100000 habitantes en países industrializados ${ }^{(4)}$, siendo en Estados Unidos 15 por cada 100000 habitantes ${ }^{(5)}$.

En un estudio multinacional en el 2009 liderado por Murdoch en 58 hospitales de 25 países, observó que la tasa de mortalidad hospitalaria fue de $15 \%$ al $20 \%$ con una mortalidad de 1 año cercana al 40\% ${ }^{(6)}$. En el año 2016, María del Cisne Rojas, desarrolló un estudio en el Hospital de las Fuerzas Armadas de Ecuador con 163 participantes, con el objetivo de determinar la etiología y factores de riesgo de la $\mathrm{El}$, concluyendo que el riesgo aumenta con la edad y prevaleciendo en mujeres, el agente etiológico aislado con mayor frecuencia fue el Streptococcus pyogenes (57\%) en pacientes con antecedentes de diabetes (63\%) e hipertensión $(60 \%)^{(7)}$. En Venezuela, Felipe Jesús y col. desarrollaron un estudio con 41 participantes en el año 2011 en el Hospital Universitario Dr. Luis Gómez López encontrando que el género masculino $(82,9 \%)$ con una edad promedio de 45 años y el agente etiológico estafilococo $(36,7 \%)$ eran los más frecuentes, los síntomas más prevalentes fueron fiebre $(80 \%)$ y disnea (56\%), la válvula aórtica fue la más afectada $(36,6 \%)$ y las complicaciones más frecuentes fueron insuficiencia renal aguda (39\%), sepsis $(21,9 \%)$ e insuficiencia cardíaca $(19,5 \%)^{(8)}$.

A nivel nacional, en el 2006 se desarrolló un estudio entre junio de 1999 y junio de 2005 en el Hospital Nacional Dos de Mayo (HNDM) que involucró a 75 pacientes con el objetivo de evaluar la evolución y letalidad de los pacientes portadores de endocarditis infecciosa, revelando una tasa de letalidad de $14,7 \%{ }^{(9)}$. El presente estudio se ha planteado en la misma institución con el objetivo de determinar las características clínicas y epidemiológicas de la endocarditis infecciosa en el Hospital Nacional Dos de Mayo en los últimos años (2014 -2019), teniendo en cuenta sus características sociodemográficas, agente etiológico, signos y síntomas, factores de riesgo, complicaciones, compromiso de válvula cardíaca, régimen antibiótico y el valor de amplitud de distribución eritrocitaria (ADE). Recientemente se ha identificado a la ADE como un marcador biológico con valor pronóstico de mortalidad y complicaciones en enfermedades cardiovasculares ${ }^{(10)}$. Los resultados de esta investigación contribuirán a caracterizar mejor esta entidad nosológica en nuestro país.

\section{MÉTODOS}

\section{Diseño y población de estudio}

Se realizó un estudio observacional, descriptivo, transversal y retrospectivo en el Hospital Nacional Dos de Mayo del Ministerio de Salud en Lima, Perú, categoría III-1.

\section{Criterios de inclusión y exclusión}

Se incluyeron a los pacientes mayores de 18 años con diagnóstico definitivo de endocarditis infecciosa según los criterios de Duke modificados, hospitalizados en el periodo 2014-2019, que contaban con hemograma, hemocultivo y ecocardiograma. Se excluyeron 7 historias clínicas sin la información necesaria.

\section{Instrumento de recolección}

Se usó una ficha de recolección como instrumento, que fue estructurada de acuerdo a las variables de interés del estudio: edad, sexo, procedencia, grado de instrucción, signos, síntomas, agen- te etiológico, complicaciones, factores de riesgo, válvula afectada, tratamiento, ADE (el valor de referencia del HN2DM fue $11,6 \%$ - $14,4 \%$ ) y mortalidad, y validada por 5 médicos especialistas.

\section{Análisis estadístico}

Los datos obtenidos fueron ingresados en el programa SPSS versión 25; para las variables cuantitativas se utilizaron medidas de tendencia central y desviación estándar, mientras que las variables cualitativas se expresaron en frecuencias y porcentajes.

\section{Consideraciones éticas}

El presente estudio fue aprobado por el Comité de Ética de la Facultad de Medicina Humana de la Universidad San Martín de Porres y del HN2DM. Los nombres de los sujetos se mantuvieron en estricta confidencialidad y posteriormente fueron codificados a fin de mantener el anonimato.

\section{RESULTADOS}

Se revisaron 31 historias clínicas, de las cuales 26 contenían las variables de estudio. La edad promedio de los pacientes fue 46,96 $\pm 15,601$ y el rango de edad fue de 18 a 72 años. El 46,2\% (n=12) fueron mayores de 50 años; la mayoría del género masculino, siendo $57,7 \% \quad(n=15)$ hombres y $42,3 \%(n=11)$ mujeres. El $57,7 \%$ ( $n=15)$ procedían de Lima, 26,9\% $(n=7)$ de la Costa (Piura, Chiclayo, Trujillo e Ica), $11,5 \%(n=3)$ de la Sierra (Apurímac, Puno y Cusco), y 3,8\% (n=1) de la Selva (Ucayali). El 50\% ( $n=13)$ tenía como grado de instrucción secundaria completa (Tabla 1).

El síntoma predominante fue fiebre con un $26 \%(n=20)$, el 23,4\% ( $n=18)$ presentó disnea, $13 \%(n=10)$ pérdida de peso, $7,8 \%(n=6)$ escalofríos, 5,2\% ( $n=4)$ dolor de espalda, 2,6\% ( $n=2)$ mialgias y artralgias, y $22,1 \%(n=17)$ otros síntomas como tos, dolor torácico y abdominal, diaforesis profusa, náuseas, vómitos, convulsiones, malestar general, palpitaciones, cefalea y sangrado nasal.

La complicación más frecuente fue la insuficiencia cardíaca con un $61 \%(n=25)$, $12,2 \%(n=5)$ presentaron infección in- 
Tabla 1. Características sociodemográficas de los pacientes con endocarditis infecciosa atendidos en el Hospital Nacional Dos de Mayo, 2014-2019, Lima- Perú.

\begin{tabular}{|c|c|c|}
\hline Características sociodemográficas & $\begin{array}{c}N=26 \\
n\end{array}$ & $\begin{array}{c}\text { Porcentaje } \\
\text { (\%) }\end{array}$ \\
\hline Edad (media-DS) & $46,96 \pm 15,601$ & \\
\hline \multicolumn{3}{|l|}{ Edad } \\
\hline$<20$ & 2 & 7,7 \\
\hline $21-30$ & 1 & 3,8 \\
\hline $31-40$ & 7 & 26,9 \\
\hline $41-50$ & 4 & 15,4 \\
\hline$>50$ & 12 & 46,2 \\
\hline \multicolumn{3}{|l|}{ Sexo } \\
\hline Masculino & 15 & 57,7 \\
\hline Femenino & 11 & 42,3 \\
\hline \multicolumn{3}{|l|}{ Procedencia } \\
\hline Lima & 15 & 57,7 \\
\hline Costa $(*)$ & 7 & 26,9 \\
\hline Sierra & 3 & 11,5 \\
\hline Selva & 1 & 3,8 \\
\hline \multicolumn{3}{|l|}{ Grado de instrucción } \\
\hline Ninguno & 2 & 7,7 \\
\hline Primaria incompleta & 1 & 3,8 \\
\hline Primaria completa & 1 & 3,8 \\
\hline Secundaria incompleta & 4 & 15,4 \\
\hline Secundaria completa & 13 & 50,0 \\
\hline Técnico superior & 4 & 15,4 \\
\hline Universitario superior & 1 & 3,8 \\
\hline
\end{tabular}

$(*)$ Excepción de Lima

controlada, $7,3 \%(n=3)$ embolias y $19,5 \%$ $(n=8)$ otras complicaciones (absceso valvular, hipertensión pulmonar, regurgitación aórtica, ACV, aneurisma del seno de Valsalva, shock séptico y parada cardiorrespiratoria) (Tabla 2).

El signo más frecuente fue el soplo cardíaco (sistólico, diastólico, holosistólico y sistodiastólico) con un $67,6 \%(n=25)$, el $8,1 \%(n=3)$ lesiones de Janeway, 2,7\% $(n=1)$ nódulos de Osler y $21,6 \% \quad(n=8)$ otros signos (edema de miembros inferiores, frémito, choque de punta, expectoración blanquecina, hemoptoica y verdosa).

En relación a los factores de riesgo se encontró que el 47,1\% ( $n=16)$ presentaron comorbilidades como infección por VIH, diabetes mellitus tipo 2, infección por virus de la hepatitis $C$, infección por virus de la hepatitis $B$, aneurisma de la arteria cubital, extracción dentaria, infección urinaria, paraplejía secuelar, úlceras por presión, mieloma múltiple, hipertensión arterial, gammapatía monoclonal, celulitis, enfermedad renal crónica, diálisis, usuario de drogas, gingivitis, caries dental, necrosis de la pulpa dentaria, ACV, periodontitis, tabaquismo, cirugía mayor y enfermedad diverticular. El 26,5\% ( $n=9)$ tuvo procedimientos de riesgo (cirugía dentaria, diálisis, cirugía gastrointestinal, parto eutócico y cesárea), 8,8\% ( $n=3)$ válvula protésica, $5,9 \%(n=2)$ cardiopatías congénitas y en el $11,8 \%(n=4)$ no se encontró ningún factor de riesgo.

Se objetivaron, según los resultados de los ecocardiogramas, la afectación de las válvulas cardíacas, el 51,7\% ( $n=15)$ correspondió a la válvula mitral, 44,8\% $(n=13)$ a la válvula aortica, y el 3,4\% $(n=1)$ válvula tricúspide (Tabla 3).

El 57,7\% ( $n=15)$ tuvieron hemocultivos negativos, $7,7 \%(n=2)$ Staphylococcus aereus, 3,8\% ( $n=1)$ S. grupo viridans, $3,8 \%(n=1)$ Enterococcus spp, y $15,4 \%$ $(n=4)$ otros agentes (Staphylococcus copitis, Acinetobacter baumanii, Enterobacter clocae y Streptococcus sp).

El 19,2\% ( $n=5)$ recibió vancomicina + ceftriaxona, 19,2\% ( $n=5)$ vancomicina + gentamicina, 7,7\% ( $n=2)$ vancomicina + meropenem, 7,7\% ( $n=2)$ vancomicina + gentamicina + ceftriaxona, 3,8\% $(n=1)$ imipenem/cilastatina + ceftriaxona, 3,8\% $(n=1)$ vancomicina, 3,8\% $(n=1)$ ceftriaxona + amikacina, 3,8\% ( $n=1)$ ceftriaxona + gentamicina y $30,8 \%(n=8)$ otros antibióticos (meropenem, ceftriaxona, ampicilina/sulbactam + gentamicina, entre otros) (Tabla 4).

En relación a la amplitud de distribución eritrocitaria, se encontró que el $88 \%(n=22)$ presentaron un rango $>14,4 \%$ y el $12 \%$ $(n=3)$ un rango de $11,6-14,4 \%$ en uno de los sujetos no se encontró el valor de ADE (Tabla 5).

Del total de pacientes ( $n=26$ ), el 38,5\% $(n=10)$ de los pacientes con El fallecieron como consecuencia de shock séptico, insuficiencia respiratoria, falla cardíaca y neumonía complicada. De los 10 pacientes fallecidos, 9 tuvieron valores de ADE > $14,4 \%$ y 1 tuvo un valor de ADE de $13,4 \%$.

\section{DISCUSIÓN}

En nuestro estudio se evidenció que la media de la edad fue de 46,96 $\pm 15,60 \%$, lo cual se asemeja a los estudios realizados a nivel nacional e internacional (11-14). La mayor parte de los casos correspondieron al sexo masculino $(57,7 \%)$ similar a lo encontrado en la bibliografía ${ }^{(11,15,16,17)}$, debido probablemente al papel protector de los estrógenos al endotelio en las mujeres en edad reproductiva ${ }^{(18)}$. En relación al agente etiológico se evidenció que el 57,7\% presentaron hemocultivo negativo debido al posible uso de antibióticos previo a la hospitalización; sin embargo, el Staphylococcus aereus $(7,7 \%)$ fue el agente causal que predominó en los pacientes con hemocultivo positivo encontrando concordancia con los estudios revisados $(11,13,16,17)$.

Según Echevarría y col., las manifestaciones clínicas evidenciadas en su estudio fueron soplos cardíacos encontrándose en un $74,4 \%$, fiebre $(71,8 \%)$ y disnea $(59,9 \%)$ (19). Según Flores y col., las manifestaciones 
Tabla 2. Síntomas y complicaciones de los pacientes con endocarditis infecciosa atendidos en el Hospital Nacional Dos de Mayo, 2014-2019, Lima- Perú.

\begin{tabular}{lcc} 
Síntomas y complicaciones & $\begin{array}{c}\mathbf{N}=\mathbf{2 6} \\
\mathbf{n}\end{array}$ & $\begin{array}{c}\text { Porcentaje } \\
\text { (\%) }\end{array}$ \\
Síntomas & 20 & 26 \\
\hline Fiebre & 18 & 23,4 \\
\hline Disnea & 10 & 13 \\
\hline Pérdida de peso & 6 & 7,8 \\
\hline Escalofríos & 4 & 5,2 \\
\hline Dolor de espalda & 2 & 2,6 \\
\hline Mialgias y artralgias & 17 & 22,1 \\
\hline Otros síntomas & & 61 \\
\hline Complicaciones & 25 & 12,2 \\
\hline Insuficiencia cardíaca & 5 & 7,3 \\
\hline Infección incontrolada & 3 & 19,5 \\
\hline Embolias & 8 & \\
\hline Otras complicaciones & & \\
\hline
\end{tabular}

clínicas encontradas fueron compromiso del estado general $(79,4 \%)$, fiebre $(71,4 \%)$ y soplo cardíaco $(65,7 \%)^{(11)}$. Salamanca $M$. encontró como hallazgos más frecuentes a la fiebre (90\%) y soplo cardíaco (45\%) ${ }^{(13)}$. En nuestro estudio, se observó fiebre en un $26 \%$, disnea $(23,4 \%)$, el signo más frecuente fue el soplo cardíaco con un 67,6\%; en menor frecuencia se encontraron las lesiones Janeway $(8,1 \%)$ y nódulos de Osler $(2,7 \%)$, estos guardan similitud con los estudios anteriormente mencionados ${ }^{(11,13,19)}$.

Las complicaciones principales fueron insuficiencia cardíaca (71\%), infección incontrolada $(12,2 \%)$ y embolia $(7,3 \%)$, esto también se evidenció en otros estudios predominando la insuficiencia

Tabla 3. Factores de riesgo y válvulas cardíacas comprometidas de los pacientes con endocarditis infecciosa atendidos en el Hospital Nacional Dos de Mayo, 2014-2019, Lima- Perú.

\begin{tabular}{lcc} 
Factores de riesgo y válvula cardiaca & $\begin{array}{c}\mathbf{N}=\mathbf{2 6} \\
\mathbf{n}\end{array}$ & Porcentaje (\%) \\
\hline Factores de riesgo & 16 & 47,1 \\
\hline Comorbilidades & 9 & 26,5 \\
\hline Procedimientos de riesgo & 3 & 8,8 \\
\hline Válvula protésica & 2 & 5,9 \\
\hline Cardiopatías congénitas & 4 & 11,8 \\
\hline Ningún factor de riesgo & & 51,7 \\
\hline Válvula cardíaca & 15 & 44,8 \\
\hline Válvula mitral & 13 & 3,4 \\
\hline Válvula aórtica & 1 & \\
\hline Válvula tricúspide & & \\
\hline
\end{tabular}

enfermedad renal crónica con hemodiálisis, diabetes mellitus, entre otros como las comorbilidades más frecuentes ${ }^{(13)}$.

En relación a los procedimientos de riesgo, se encontró a la extracción dentaria $(n=5)$ como el más predominante, seguido de cesárea $(n=2)$, cirugía gastrointestinal $(n=2)$, parto eutócico, traumatismo de cadera izquierda y diálisis, siendo 1 caso en cada uno de los últimos 3. No se realizó la comparación con otros estudios, ya que no describieron los procedimientos de riesgos.

Se demostró que las válvulas protésicas afectaron a 3 pacientes $(8,8 \%)$, a diferencia del estudio de Flores P. donde se encontraron a 10 pacientes $(28,6 \%)^{(11)}$ y del estudio de Rubio L. constituyendo 14 pacientes (25\%) portadores de válvula protésica con diagnóstico de El ${ }^{(16)}$.

Con respecto al compromiso de las válvulas cardíacas, en nuestro estudio se evidenció que la válvula mitral $(51,7 \%)$ fue la más afectada, seguido de la válvula aórtica (44,8\%), resultado que se asemeja al estudio de Salamanca $M$. donde se encontraron 8 pacientes $(40 \%){ }^{(13)}$, a diferencia del estudio de Flores P. donde la válvula aórtica fue la más comprometida $(54,3 \%)^{(11)}$. Asimismo, en el estudio de Rubio L. se presentó en $(46,4 \%)^{(16)}$. En el estudio de Guray Y. se presentó en un $(39 \%)^{(15)}$, los resultados de ese estudio no mostraron una diferencia significativa respecto al compromiso de ambas válvulas, concluyendo que en la El las válvulas que tienen mayor compromiso son la mitral y aórtica.

La El es una enfermedad poco frecuente, pero con una alta mortalidad. Este estudio contó con 26 pacientes, 10 fallecieron, de los cuales 9 tuvieron valores de $A D E>14,4 \%$ y 1 tuvo un valor de ADE en el rango de normalidad (11,6 - 14,4\%); 25 presentaron complicaciones de los cuales 22 presentaron valores $>$ $14,4 \%$, lo que nos confirmaría la elevada mortalidad de esta enfermedad y la relación entre los valores elevados del ADE (> $14,4 \%)$ con la presencia de complicaciones y mortalidad. Las principales causas de mortalidad en nuestro estudio fueron shock séptico $(n=4)$, falla cardíaca $(n=4)$ e insuficiencia respiratoria $(n=3)$, resultados similares a los estudios de Sánchez E. 
Tabla 4. Agente etiológico y régimen antibiótico en los pacientes con endocarditis infecciosa atendidos en el Hospital Nacional Dos de Mayo, 2014-2019, Lima- Perú

\begin{tabular}{|c|c|c|}
\hline Agente etiológico y régimen antibiótico & $\begin{array}{c}N=26 \\
n\end{array}$ & $\begin{array}{c}\text { Porcentaje } \\
\text { (\%) }\end{array}$ \\
\hline \multicolumn{3}{|l|}{ Agente etiólogico } \\
\hline Hemocultivo negativo & 15 & 57,7 \\
\hline Staphylococcus aereus & 2 & 7,7 \\
\hline S. grupo viridans & 1 & 3,8 \\
\hline Enterococcus spp & 1 & 3,8 \\
\hline Otros & 4 & 15,4 \\
\hline \multicolumn{3}{|l|}{ Régimen antibiótico } \\
\hline Vancomicina + ceftriaxona & 5 & 19,2 \\
\hline Vancomicina + gentamicina & 5 & 19,2 \\
\hline Vancomicina + meropenem & 2 & 7,7 \\
\hline Vancomicina + gentamicina + ceftriaxona & 2 & 7,7 \\
\hline Imipenem/cilastatina + ampicilina & 1 & 3,8 \\
\hline Vancomicina & 1 & 3,8 \\
\hline Ceftriaxona + amikacina & 1 & 3,8 \\
\hline Ceftriaxona + gentamicina & 1 & 3,8 \\
\hline Otros & 8 & 30,8 \\
\hline
\end{tabular}

realizado en el 2006, donde se evidenció una tasa de letalidad de $14,7 \%{ }^{(9)}$ y de Polo G. y col. en el 2020 con una mortalidad de 20,3\%, donde el shock séptico fue la principal causa de muerte ${ }^{(20)}$. En nuestro estudio se encontró una mayor mortalidad a comparación de otros estudios, esto probablemente a que existen diferencias respecto al tamaño y características de la muestra; así, en el estudio de Sánchez E. realizado en un periodo 7 años (1999 a 2005), en la misma institución en que se realizó esta investigación, incluyeron menores de 18 años y encontraron 75 sujetos con El, de los cuales el 76\% tuvieron cardiopatía reumática ${ }^{(9)}$ que les generaba un factor predisponente para El, según nuestros resultados en un periodo de 6 años se encontraron sólo 26 casos de El, esto probablemente se deba a que

Tabla 5. Amplitud de distribución eritrocitaria (ADE) de los pacientes con endocarditis infecciosa atendidos en el Hospital Nacional Dos de Mayo, 2014-2019, Lima-Perú.

\begin{tabular}{lcc} 
ADE & $\mathbf{N}=\mathbf{2 6}$ & $\begin{array}{c}\text { Porcentaje } \\
\mathbf{( \% )}\end{array}$ \\
\hline $11,6-14,4 \%$ & 3 & 12 \\
\hline$>14,4 \%$ & 22 & 88 \\
\hline Total & 25 & 100 \\
\hline
\end{tabular}

ADE: amplitud de distribución eritrocitaria la fiebre reumática ha disminuido su prevalencia en los últimos años.

Respecto al tratamiento, la mayor parte de pacientes recibió la combinación de vancomicina + ceftriaxona $(19,2 \%)$ y vancomicina + gentamicina (19,2\%). Comparando estos resultados con las actuales guías de manejo de la endocarditis infecciosa, encontramos que hay coincidencias en cuanto al manejo empírico con ambos tratamientos ${ }^{(3)}$.

Nuestro estudio tuvo limitaciones tales como: un tamaño muestral reducido que dificultó realizar comparaciones con investigaciones de mayor población, haberse realizado en un solo centro hospitalario, ser un estudio retrospectivo, algunas historias clínicas estaban incompletas y hemocultivos negativos debido al uso de antibióticos previo al ingreso hospitalario. Como fortalezas consideramos a este estudio innovador por la relación del ADE con la El como un marcador de mortalidad y complicaciones con valores elevados (> $14,4 \%)$, sirviendo como base para futuras investigaciones sobre el tema.

Concluimos, según nuestros hallazgos, que la El afecta principalmente a mayores de 50 años, de sexo masculino, la sintomatología más frecuente fue soplo cardíaco $(67,6 \%)$, fiebre $(26 \%)$ y disnea $(23,4 \%)$, la complicación predominante fue la insuficiencia cardíaca (61\%). Las comorbilidades $(47,1 \%)$ fueron el principal factor de riesgo. La válvula mitral (51,7\%) fue la más comprometida. Más de la mitad tuvieron hemocultivo negativo. El régimen antibiótico vancomicina + ceftriaxona y vancomicina + gentamicina fueron los más usados. Un 88\% presentaron $\mathrm{ADE}>14,4 \%$. De 10 pacientes que fallecieron, 9 tuvieron ADE > 14,4\%, lo cual reafirma a este marcador biológico como un predictor pronóstico en enfermedades cardiovasculares.

Se recomienda realizar más estudios sobre la Endocarditis infecciosa que se relacionen a marcadores de mortalidad como el ADE, así como estudios multicéntricos para representar la situación epidemiológica de la El en nuestro país.

\section{AGRADECIMIENTOS}

A Susel Espinoza y Shakira Tafur por el apoyo en la búsqueda bibliográfica relacionada al tema de esta investigación.

\section{REFERENCIAS BIBLIOGRÁFICAS}

1. Deloya A, López M, Reyna J, Saturno G. Guía de práctica clínica en endocarditis infecciosa aguda y subaguda. México: Secretaría de Salud; 2010.

2. Romero J, Aveiro A. Características clínicas, bacteriológicas y demográficas de las endocarditis infecciosas. Rev Nac. 2020;12(1). DOI: 10.18004/ rdn2020.0012.01.042-054

3. Habib G, Lancellotti P, Antunes M, Bongiorni M Casalta J, del Zotti F, et al. Guia ESC 2015 sobre el tratamiento de la endocarditis infecciosa. Rev Esp Cardiol. 2016;69(1):69.e1-e49. DOI: 10.1016/j. recesp.2015.12.001

4. Santaularia M, Vega Á, Pérez D. Endocarditis infecciosa. Evid Med Invest Salud. 2014;7(2):76-83.

5. Wang A, Gaca J, Chu V. Management Considerations in Infective Endocarditis. JAMA. 2018;320(1):102. DOI: 10.1001/jama.2018.7596

6. Murdoch D, Corey G, Hoen B, Miró J, Fowler V, Bayer AS, et al. Clinical Presentation, Etiology, and Outcome of Infective Endocarditis in the 21st Century The. Arch Intern Med. 2009;169(5):463. DOI: 10.1001/archinternmed.2008.603

7. Rojas M. Etiologia y Factores de riesgo de la Endocarditis Infecciosa en el Hospital de las Fuerzas Armadas №1 durante el periodo 2013-2015 (Tesis de Bachiller). Loja-Ecuador: Universidad Nacional de Loja. 2016.

8. Felipe J, López G, Jesús K, Higuera A, Mujica M. Caracteristicas clinicas y epidemiológicas de la 
endocarditis infecciosa en pacientes admitidos en el servicio de cardiología en el Hospital universitario Dr. Luis Gómez López. Barquisimeto, estado Lara; 2011.

9. Sánchez E, Gutiérrez C. Evolución y letalidad por endocarditis infecciosa en pacientes atendidos en el Hospital Nacional Dos de Mayo. Anales de la Facultad de Medicina. 2006;67(2):125-133.

10. Alcaíno H, Pozo J, Pavez M, Toledo H. Ancho de distribución eritrocitaria como potencial biomarcador clínico en enfermedades cardiovasculares. Rev Med Chile. 2016;144: 634-642.

11. Flores P, González N, Betancourt P, Berho J, Astudillo C, García C, et al. Endocarditis infecciosa: caracterización clínica de la enfermedad. Revisión de casos de los últimos 5 años. Rev Chil Cardiol. 2017;36(1):34-40. DOI: 10.4067/S071885602017000100004.

12. Wei X, Liu Y, He P, Zhou Y, Tan N, Chen J, et al. Combined efficacy of $\mathrm{C}$-reactive protein and red blood cell distribution width in prognosis of patients with culture-negative infective endocarditis. Oncotarget. 2017;8(41):71173-80. DOI: 10.18632/ oncotarget.16888.

13. Salamanca M. Perfil clínico y epidemiológico de la endocarditis infecciosa en el Hospital Naciona Guillermo Almenara Irigoyen de Lima durante un periodo de 10 años, 2005 - 2014 (Tesis de Bachiller). Tacna-Perú: Universidad Nacional Jorge Basadre Grohman. 2015.

14. Sénior J, Gándara J. Endocarditis infecciosa. latreia. 2015; 28(4):456-71. DOI: 10.17533/udea.iatreia. v28n4a11

15. Guray Y, Ipek E, Guray U, Demirkan B, Kafes $H$, Asarcikli LD, et al. Red cell distribution width predicts mortality in infective endocarditis. Arch Cardiovasc Dis. 2014 May;107(5):299-307. DOI: 10.1016/J.ACVD.2014.04.008

16. Rubio L. Perfil clínico, espectro microbiológico, evolución y factores pronósticos en pacientes con endocarditis infecciosa (Tesis de Bachiller). LimaPerú: Instituto Nacional del Corazón. 2009.

17. Olaya A, Vargas D, Montes L, Avila Y, Carcamo L. Descripción clínica, epidemiológica y ecocardiográfica de la endocarditis infecciosa. Acta med. colomb. 2019:44(4). DOI: 10.36104/amc.2019.1223

18. Olivera C, Lubián S. Endocarditis infecciosa tratamiento y profilaxis. Protocolos Diagnósticos y Terapéuticos en Cardiologia Pediátrica, Hospital Universitario Puerta del Mar. Cádiz, España. 2005: 1-14.

19. Echevarria G, Echevarria S, Eguren O, Fierro L. Características clínicas y epidemiológicas de la endocarditis infecciosa en el INSN (Tesis de Bachiller). Lima-Perú: Instituto Nacional de Salud del niño. 2011.

20. Polo G, Torres L, Yarahuaman J, Lobato C, Uribe E. Experiencia de cinco años en el manejo de endocarditis infecciosa complicada en un centro de referencia nacional. Archivos Peruanos De Cardiologia Y Cirugia Cardiovascular. 2020;1(3):172-7. DOI: 10.47487/APCYCCV.V1I3.77 